\title{
Right Here, Right Now, on Purpose
}

\author{
Amelia Wendt ${ }^{1}$ (D)
}

Received: 6 October 2020 / Accepted: 27 October 2020 / Published online: 16 November 2020

(C) Academic Psychiatry 2020

My mind was racing as I stood in the COVID-19 screening line. It had started off as a normal weekday - well, "normal" in the context of 2020. My mind jumped from one crisis to another, the worldwide pandemic, police brutality, racial injustice, and the upcoming election. I looked up at the sky and saw it filled with smoke, reminders of the wildfires burning across the Western United States. I closed my eyes and felt the same smoke hanging heavily, clouding my attention. With these thoughts spinning, I completed my screening and rushed in to my office.

Today, I was scheduled to attend a mindfulness group through my clinical rotation at the Veterans Administration. I logged on to join the virtual group room and was greeted by a number of veterans and the group facilitator. The veterans were starting their weekly check-in, sharing the number of times and situations they had practiced mindfulness over the past week.

Every night before bed.

During a Zoom break in the middle of the day.

To help stay sober.

When my back pain was really bad.

After this check-in, each veteran shared what mindfulness meant to them and what it looked like in their lives.

It can be anything - even washing the dishes or going for a walk.

Even in 5 minutes I can do it.

Your breath - it's with you wherever you go.

It's being right here, right now, on purpose.

I joined the group for a mindfulness exercise. We slowed our breath, noticed our thoughts, and gently brought our minds back to the present. With my eyes closed, I noticed the haze obscuring the present moment start to lift. As my attention shifted to the here and now, I felt the spinning crises slow to a rest in the past and the future. I felt my heart rate slow, my shoulders relax, and my mind quiet. I was surprised to notice the tears behind my closed eyes. I was right here, right now, on purpose. We opened our eyes and shared what we noticed.

I noticed how my mind wandered, and that's ok.

Because it's a practice, not a perfection.

Isn't that amazing...

I was trying to change my thoughts, and then I thought, wait a minute, that's not what we're supposed to do! So I just tried to focus on my breathing, and that's all I can do.

The brain doesn't get some rest until we give it some rest. Thank you for that rest.

I realized that this hour was the longest amount of time I had slowed down in weeks - or maybe even months. Not responding to emails, scrolling through my phone, listening to the news, watching another show on Netflix. These veterans were teaching me just how powerful that slowing down can be.

I went about the rest of my workday. At the end of the day, I stepped out of the VA, looked up at the sky, and noticed a small patch of blue peering through the smoke-filled haze. I took a deep breath and slowly exhaled.

I was right here, right now, on purpose.

\section{Compliance with Ethical Standards}

Disclosures The author states that there is no conflict of interest.

Ethical Considerations The identities of the subjects in the essay are masked.

Publisher's Note Springer Nature remains neutral with regard to jurisdictional claims in published maps and institutional affiliations.
Amelia Wendt

wendt616@uw.edu

1 University of Washington School of Medicine, Seattle, WA, USA 